Pacific Journal of Mathematics

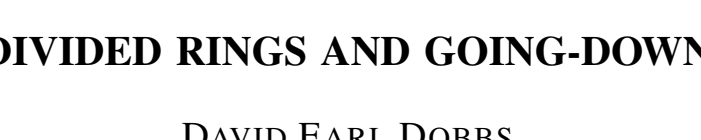




\section{DIVIDED RINGS AND GOING-DOWN}

\section{DAVID E. DoBbS}

Going-down rings are characterized in terms of the $A \mathrm{~V}$ domains of Akiba. As a result, the internal characterization of going-down rings which has recently been established by McAdam in the integrally closed case is generalized to the root-closed case, but is shown to fail in general.

1. Introduction. Let $R$ be a (commutative integral) domain. As in [5] and [6], $R$ is called a going-down ring (and we write: $R$ is $G D$ ) in case $R \subset S$ satisfies going-down for each domain $S$ containing $R$; by [6, Theorem 1], the test domains $S$ considered may be restricted either to be valuation overrings of $R$ or to be simple overrings of $R$. The present paper contributes to the search for an internal characterization of going-down rings; attention may be focused on the quasi-local case, as being $G D$ is a local property (cf. [5, Lemma 2.1]).

The domain $R$ is said to be divided in case each $P$ in $\operatorname{Spec}(R)$ satisfies $P=P R_{P}$; that is, if each element of $R \backslash P$ divides each element of $P$, with quotient in $R$. (The preceding definition is motivated by considering valuation domains, perhaps the most significant type of quasilocal going-down rings. Divided rings are the $A V$-domains studied by Akiba [1].) In [14, Corollary 11], McAdam established that, for quasi-local integrally closed $R$, being $G D$ is equivalent to being divided. The equivalence is extended to the case of quasilocal root-closed $R$ (Corollary 2.8) as a consequence of our main result (Theorem 2.5): a quasi-local domain $R$ is $G D$ if and only if $R$ has a divided integral overring $T$ (for which the contraction map $\operatorname{Spec}(T) \rightarrow \operatorname{Spec}(R)$ is necessarily a homeomorphism).

Theorem 2.5 is best-possible, for while divided implies quasi-local $G D$ (Proposition 2.1), analysis of a recent example of Boisen and Sheldon [3] in Example 2.9 shows that the converse is false. Some sufficient conditions for the validity of the converse are obtained in Proposition 2.10 in terms of the overrings $T$ guaranteed by Theorem 2.5; these, in turn, give new information about the example in [3]. Remark 2.11 generalizes some work of Papick [15], by illustrating how Theorem 2.5 may be employed to infer results about goingdown rings from corresponding facts about divided rings. A related external characterization of going-down rings and alternate methods are treated in the brief final section.

Throughout, $R$ denotes a domain. Unexplained terminology is standard, as in [8] and [11]. 
2. Divided extensions, Recall from [5, Theorem 2.2] that a going-down ring must be treed, in the sense that no maximal ideal contains incomparable prime ideals. As W. J. Lewis has constructed a treed domain which is not $G D$ [16, Example 6.4], our first result generalizes [1, Theorem 1] and [12, p. 59].

Proposition 2.1. Any divided domain is quasi-local and GD.

Proof. Let $R$ be divided. Any prime ideal $P$ of $R$ satisfies $P=P R_{P}$ and, thus, is comparable to any ideal of $R$ (cf. proof of [1, Theorem 1] and [9, Proposition 1.2 (ii)]; in particular, $R$ is quasilocal. If the assertion fails, the characterization of going-down in [11, Exercise 37(iii), p. 44] yields a domain $T$ containing $R, P$ in Spec $(R)$, and $Q$ minimal amongst primes of $T$ containing $P T$, such that $P T \cap(R \backslash P)(T \backslash Q) \neq \varnothing$. Then $\Sigma p_{i} t_{i}=r t$, with $p_{i}$ in $P, t_{i}$ in $T, r$ in $R \backslash P$, and $t$ in $T \backslash Q$. As $R$ is divided, $r_{i}=p_{i} r^{-1}$ is in $P$ so that $t=\Sigma r_{i} t_{i}$ is in $P T \subset Q$, the desired contradiction, to complete the proof.

It is convenient next to record three elementary facts about divided rings. The analogous statements about going-down rings are in [5, Lemma 2.1]; [6, Corollary]; and Remarks 2.11 and 3.2(a), (b), respectively.

Lemma 2.2. (a) Any localization of a divided domain is divided.

(b) Let $K+M$ be a valuation domain with maximal ideal $M$, where $K$ is a field; let $D$ be a subring of $K$. Then $D+M$ is divided if and only if $D$ is divided.

(c) (Cf. [1, Theorem 3].) Let $R$ be divided. For any ideal I of $R$, each prime ideal of $R / I$ is comparable to each ideal of $R / I$. In particular, for $P$ in $\operatorname{Spec}(R), R / P$ is divided.

Proof. All parts are straightforward. We give the proof for (a). (The proof of a special case of it in [1, Proposition 3] contains a trivial misprint. In fact, " $R_{p}=\left(R_{p}\right)_{Q_{p}}$ " should read " $R_{Q}=$ $\left.\left(R_{p}\right)_{Q R_{p}}\right)$." Let $S$ be a multiplicatively closed subset of a divided domain $R$. It suffices to shown that, if $Q \in \operatorname{Spec}\left(R_{S}\right)$ and $b \in R_{S} \backslash Q$, then $Q \subset R_{S} b$. As $Q=P R_{S}$ with $P=Q \cap R$ in $\operatorname{Spec}(R)$, it is enough to show $P \subset R b$; this follows since $R$ is divided and we may assume that $b \in R \backslash P$, proving (a).

Part (b) is an easy consequence of [8, Theorem A (c), (d), (e), (g), p. 560]. We may safely omit the proof of (c). 
The next result is essentially known, but is recorded here for lack of a convenient reference. First, recall that an extension $A \subset B$ of commutative rings is called unibranched in case the contraction map $\operatorname{Spec}(B) \rightarrow \operatorname{Spec}(A)$ is a bijection; in that case, $A$ being understood, we shall often term $B$ "unibranched."

LEMMA 2.3. Let $R \subset T$ be an integral unibranched extension of domains. Then $R$ is $G D$ if and only if $T$ is $G D$.

Proof. For the "if" half, assume that $T$ is $G D$, apply [7, Lemma 2.2] to show that $R \subset T$ satisfies going-down, and then argue as in either [13, Theorem 1] or [7, Theorem 2.4] to conclude that $R$ is GD. Conversely, the "only if" half is a special case of [15, Proposition 2.12].

Prior to stating our main result, we isolate a technical lemma. It will be convenient to say that a prime ideal $P$ of $R$ is divided in $R$ if $P=P R_{P}$; otherwise, $P$ is said to be nondivided in $R$. Note that $R$ is divided if and only if each prime of $R$ is divided in $R$. We leave to the reader the reformulations of Proposition 2.1 and Lemma 2.2 involving the "divided in" concept. As B. Greenberg has pointed out to the author, it is possible for a domain $R$ to have prime ideals $Q \subset P$, with $P$ nonmaximal and divided in $R$ and $Q$ nondivided in $R$. An explicit example of such (quasi-local pseudoBézout) $R$ has been constructed by Greenberg by modifying the material surrounding [9, Proposition 3.2].

Lemma 2.4. Let $R$ be quasi-local and $G D$, let $P \in \operatorname{Spec}(R)$ and set $T=R+P R_{P}$. Then:

(a) $R \subset T$ is integral and unibranched.

(b) If $Q$ is divided in $R$ and $Q \subset P$, then $Q$ is the prime of $T$ which contracts to $Q$ and $Q$ is divided in $T$.

(c) $P R_{P}$ is divided in $T$.

Proof. (a): For the integrality assertion, it is enough to prove that any nonzero $v$ in $P R_{P}$ is integral over $R$. We proceed via a proof modelled after that of [5, Theorem 2.2]. Express $v$ as $a b^{-1}$, with $a$ in $P$ and $b$ in $R \backslash P$. If $P$ survives in $R\left[v^{-1}\right]$, the fact that $R \subset R\left[v^{-1}\right]$ satisfies going-down, supplies a prime $W$ of $R\left[v^{-1}\right]$ which contracts to $P$; as $b=a v^{-1}$ is then in $P R\left[v^{-1}\right]$, it follows that $b$ is in $W \cap R=P$, contradiction. Thus, 1 is in $P R\left[v^{-1}\right]$; that is,

$$
1=p_{0}+p_{1} v^{-1}+\cdots+p_{n} v^{-n}
$$

for elements $p_{\imath}$ of $P$. Multiplication by $v^{n}\left(1-p_{0}\right)^{-1}$ produces the 
desired integrality equation for $v$ over $R$.

For the second assertion in (a), let $Q \in \operatorname{Spec}(R)$. As $R$ is quasilocal and treed, either $Q \varsubsetneqq P$ or $P \subset Q$. In the first case, $Q R_{P}$ is a prime of $T$ contracting to $Q$. By the incomparability property for integral extensions, it is enough to prove that any prime $I$ of $T$ which contracts to $Q$ must contain $Q R_{P}$; this, in turn, follows since $\left(Q R_{P}\right)\left(P R_{P}\right) \subset I$ and $P R_{P} \not \subset I$. For the case $P \subset Q$, note similarly that any prime of $T$ which contracts to $Q$ must contain $\left(P R_{P}\right)^{2}$ and, hence, coincides with $Q+P R_{P}$.

(b) and (c): Both admit computational proofs; we include only the details for (b). Suppose $Q$ is divided in $R$ and $Q \subset P$. By the proof of (a), $Q R_{P}$ is the prime of $T$ contracting to $Q$; as $Q$ is divided in $R, Q R_{P}=Q$. A typical element of $Q T_{Q}$ is $x=\alpha\left(r+p y^{-1}\right)^{-1}$, with $\alpha$ in $Q, y$ in $R \backslash P, r$ in $P$, and $p$ in $P$, such that $r+p y^{-1} \notin Q$. As $\left(r+p y^{-1}\right) y \in R \backslash Q$, it follows that $x=a y(r y+p)^{-1}$ is in $Q R_{Q}=Q$, to complete the proof.

Observe, as a consequence of [1, Theorem 1] and [16, Proposition 3.38], that if $R \subset T$ is an integral extension of quasi-local domains and $T$ is divided, then $R \subset T$ is unibranced; indeed, Spec $(T) \rightarrow \operatorname{Spec}(R)$ is a homeomorphism of Zariski topologies in this case. We may now present the main result.

THEOREM 2.5. For quasi-local $R$, the following are equivalent:

(a) $R$ is $G D$;

(b) $R$ has a divided integral (necessarily unibranched) overring;

(c) $R$ has a divided integral (necessarily unibranched) extension.

Proof. The implication $(b) \Rightarrow$ (c) is trivial, while $(\mathrm{c}) \Rightarrow$ (a) follows from Proposition 2.1 and Lemma 2.3. Finally, to show $(a) \Rightarrow(b)$, let $R$ be $G D$. If $R$ has finite Krull dimension, it suffices to work with nondivided primes of minimal height, while iterating the construction in Lemma 2.4. For the general case, let $F$ be the set of integral unibranched overrings of $R$; partially order $F$ by inclusion. (Note $R \in F$.) The union of any chain in $F$ is, of course, integral over $R$ and, by the proof of [1, Proposition 4], unibranched as well. Zorn's Lemma thus produces a maximal element, $T$, of $F$. If $Q$ is nondivided in $T$, then Lemma 2.4 shows that $T+Q T_{Q}$ is integral and unibranched over $T$, and hence over $R$, contradicting maximality in $F$. Therefore, $T$ is divided, as desired.

The next corollary paves the way for Corollary 2.8, another generalization of McAdam's result. As usual, $\operatorname{rad}_{S}(J)$ will denote the radical of an ideal $J$ in $S$. 
CoRollary 2.6. Let $R$ be quasi-local and GD, and let $P$ be nondivided in $R$. Then, for each $v \in P R_{P} \backslash P$, there exists $n \geqq 2$ such that $v^{n} R[v] \subset P$. Moreover, there exists $w \in P R_{P} \backslash P$ such that $w^{n} \in P$ for all $n \geqq 2$.

Proof. Let $v \in P R_{P} \backslash P$. By Lemma 2.4 or Theorem 2.5, $R$ has an integral unibranched overring $T$ such that $P R_{P}$ is contained in the prime of $T$ which contracts to $P$. Then, $S=R[v]$ is also integral and unibranched over $R$, hence quasi-local and $G D$ (by Lemma 2.3). Moreover, $v \in Q$, the prime of $S$ contracting to $P$. Let $J$ be the conductor $(R: S)=\{r \in R: r S \subset R\}$. As $S$ is quasi-local and treed, $I=\operatorname{rad}_{S}(J)$ is a prime which compares with $Q$. Since $v=a b^{-1}$ with $a \in P$ and $b \in R \backslash P$, some power of $b$ is in $J$; that is, $b \in I$, and so $I \not \subset Q$. Hence $Q \subset I$, giving $n \geqq 2$ such that $v^{n} \in J$, as required. Finally, note that if $m \geqq 2$ is minimal such that $v^{m} \in J$, then $w=v^{m-1} \in P R_{P} \backslash P$ satisfies $w^{n} \in P$ for all $n \geqq 2$.

REMARK 2.7. (a) Lemma 2.4 easily implies the following useful extension of McAdam's result. Let $R$ be quasi-local and GD. If the maximal ideal $M$ of $R$ satisfies $M R^{\prime} \subset R$, where $R^{\prime}$ denotes the integral closure of $R$, then $R$ is divided. An example of such $R$ which is not integrally closed may be obtained from a nontrivial algebraic field extension $k \subset K$ by constructing a valuation domain $K+M$ and setting $R=k+M$.

(b) Call $R$ locally divided if $R_{M}$ is divided for each maximal ideal $M$ of $R$. In that case, $R_{P}$ is divided for each $P \in \operatorname{Spec}(R)$ : the verification follows readily from the observation that $\left(R_{P}\right)_{Q R_{P}}=R_{Q}$ for primes $Q \subset P$ of $R$ [4, Propositions 7(i)-8, pp. 85-86]. One may use Lemma 2.2(a) to verify that any localization of a locally divided domain is itself locally divided; in like manner, parts (b) and (c) of Lemma 2.2 each admit "locally divided" analogues. As GD is a local property, Proposition 2.1 implies that any locally divided domain is $G D$.

(c) As in [17, p. 231], the root-closure of $R$ in an extension $T$ is $\left\{t \in T: t^{n} \in R\right.$ for some $\left.n \geqq 1\right\} ; R$ is called root-closed if the rootclosure of $R$ (in the quotient field of $R$ ) is $R$ itself. $R$ is root-closed if and only if $R_{M}$ is root-closed for each maximal ideal $M$ of $R$. If $E$ is the root-closure of a domain $D$ in a field $K$ and $V=K+M$ is a valuation domain with maximal ideal $M$, then $E+M$ is the root-closure of $D+M$. While any integrally closed domain is rootclosed, an example, $R$, illustrating failure of the converse may be obtained from a finite Galois field extension $k \subset K$ of characteristic zero with simple nonabelian Galois group and a valuation ring $K+M$, by setting $R=k+M$; that $k$ is the root-closure of $k$ in $K$, follows 
from the standard Galois theory of solvability by radicals.

(d) A most interesting class of going-down rings is that of $i$ domains [7, Corollary 2.5]. While characterizations abound [15, Corollary 2.11, Proposition 2.34], the important facts to recall for our purposes are that being an $i$-domain is a local property [16, Corollary 2.6]; and that a quasi-local domain is an $i$-domain if and only if its integral closure is a valuation domain [15, Corollary 2.15]. Note that the rings $R$ constructed in (a) and (c) above are both dividided $i$-domains. To obtain an example of a quasi-local rootclosed domain, $A$, which is neither integrally closed nor an $i$-domain, let $k \subset K$ be as in (c), let $F$ be a nontrivial purely transcendental field extension of $K$, construct a valuation domain $F+N$ with maximal ideal $N$, and set $A=k+N$. By Lemma 2.2(b), $A$ is divided; Corollary 2.8 shows this to be the normal state of affairs for quasi-local, root-closed going-down rings. However, the rings $R$ of (a) and (c) are atypical of going-down $i$-domains, by virtue of the nondivided ring in Example 2.9.

CoROLlaRy 2.8. Let $R$ be root-closed. Then $R$ is GD if and only if $R$ is locally divided.

Proof. The "if" half is contained in Remark 2.7(b). For the "only if" half, combine Corollary 2.6 with Remark 2.7(c).

EXAMPLE 2.9. We next produce, for each number $n \geqq 2$, a quasilocal going-down domain $D$ of Krull dimension $n$, which is not divided. In view of [6, Corollary], Lemma 2.2(b) and [8, Theorem $A(f)$, p. 561], the " $D+M$ construction" reduces the problem to the case $n=2$. For this, we make use of a ring introduced by Boisen and Sheldon [3, Example 1.6] for other purposes. Explicitly, let $X$ and $Y$ be algebraically independent indeterminates over a field $F$, and note that the subset $T_{1}=\left\{X^{n}: n \geqq 0\right\} \cup\left\{Y^{k} X^{m}\right.$ : $k \geqq 1, m \in Z\}$ of $F(X, Y)$ is linearly independent over $F$. The ring $D_{1}$ is taken as the $F$-span of $T_{1}$; the elements with vanishing constant term (the term involving the generator $X^{0}$ ) form a maximal ideal, $M_{1}$, of $D_{1}$. It is shown in [3, Lemma 1.7] that $V=\left(D_{1}\right)_{M_{1}}$ is a valuation domain of rank 2. If $D_{0}$ is taken as the $F$-span of

$$
T_{0}=\left\{X^{n}: n \geqq 0\right\} \cup\left\{Y^{k} X^{m}: k \geqq 1, m \geqq-k^{2}\right\},
$$

the required ring $D$ is the localization of $D_{0}$ at its maximal ideal $M_{0}=M_{1} \cap D_{0}$; by [3, Lemma 1.8] $V$ is integral over $D$.

First, observe that $D$ is an $i$-domain. (In fact, its integral closure is $V$, since $V=\left(D_{1}\right)_{D_{0} \backslash M_{0}}$ is inside the quotient field of $D$, the 
relevant calculation being $Y^{k} X^{m}=\left(Y^{k} X^{-k^{2}}\right)\left(X^{-m-k^{2}}\right)^{-1}$.) Thus, by [7, Corollary 2.3 or Theorem 2.4 or Corollary 2.5], $D$ is (quasi-local and) $G D$.

Next, to show that $D$ is not divided, recall that the nonzero primes of $D$ are, as noted in [3, Proposition 1.9], $P=\left(\left\{Y^{n} X^{-n^{2}}: n \geqq 1\right\}\right)$ and $(X)+P$. We claim that $P$ is nondivided in $D$, as $Y X^{-2} \in P D_{p} \backslash P$. Indeed, it is clear that $Y X^{-2}=\left(Y X^{-1}\right) X^{-1} \in P D_{P}$. Moreover, if $Y X^{-2}$ were expressible as a fraction of elements of $D_{0}$ with denominator having nonzero constant term, the $F$-linear independence of $T_{0}$ would lead to $\left(Y^{k} X^{m}\right) X^{2}=Y$ with $\left(k \geqq 1\right.$ and) $m \geqq-k^{2}$ and, thence, to the absurdity $-2 \geqq-1$. This establishes the claim. (Note that, as Corollary 2.6 predicts, there exists $w \in P D_{P} \backslash P$ with $w^{n} \in P$ for all $n \geqq 2: Y X^{-2}$ is such an element.)

We make three further comments about the above construction. First, recall that a domain $R$ is called Archimedean [17, p. 234] in case $\cap\left\{R r^{n}: n \geqq 1\right\}=0$ for each nonunit $r \in R$. By Corollary 2.8 and the reasoning in [2, Proposition 3.5], a quasi-local root-closed domain has Krull dimension at most 1 if and only if it is both Archimedean and GD. The "root-closed" hypothesis cannot be deleted, as $D$ is Archimedean [3, Proposition 1.10].

Second, in order to motivate Proposition 2.10, observe that $D_{P}$ is integrally closed. Indeed, it is enough to show $\left(D_{1}\right)_{D_{0} \backslash M_{0}} \subset D_{P}$. For this, we need only note that $D_{1} \subset D_{P}$, the relevant calculation being $Y^{k} X^{m}=\left(Y^{k} X^{-m}\right)\left(X^{-2 m}\right)^{-1}$ for $m<-k^{2}$.

Third, Proposition 2.10 yields the following additional information. Each nonunit of $V$ has a power lying in $D$ (see [3, p. 336, l.-4] for the corresponding assertion for certain monomials); and neither $V$ nor its maximal ideal is a finitely generated $D$-module.

In spite of Example 2.9, we next turn to ways of using Theorem 2.5 to produce conditions guaranteeing that certain quasi-local goingdown rings are divided. As usual, ht and dim will denote height and Krull dimension, respectively.

Proposition 2.10. Let $P \in \operatorname{Spec}(R)$ with $R_{P}$ integrally closed, and let $T$ be a divided integral (unibranched) overring of $R$. Then:

(a) The prime of $T$ contracting to $P$ is $P R_{P}$.

(b) If $T$ is finitely generated as an $R$-module, then $P$ is divided in $R$.

(c) Assume $\operatorname{dim}(R)=2$ and $\operatorname{ht}(P)=1$. Then, for each finite 
subset $\left\{v_{1}, \cdots, v_{m}\right\}$ of the maximal ideal $N$ of $T$, there exists $n \geqq 2$ such that $I=\Sigma R v_{i}$ satisfies $I^{n} R\left[v_{1}, \cdots, v_{m}\right] \subset R$. If, in addition, $N$ is finitely generated as an $R$-module, then $R$ is divided.

Proof. (a): If $Q$ denotes the (divided) prime of $T$ contracting to $P$, then [8, Theorem 9.11] gives $T_{R \backslash P}=T_{Q}$. As $T_{R \backslash P}$ is integral over $R_{P}$, which is integrally closed, $R_{P}=T_{Q}$. Equating maximal ideals yields $P R_{P}=Q T_{Q}=Q$.

(b): As $T \subset R_{P}$, the finite generation of $T$ implies that the conductor $(R: T)$ is not contained in $P$; consequently, there exists $r$ in $(R: T) \backslash P R_{P}$. Since $P R_{P}$ is divided in $T$, it follows that $P R_{P}=$ $r\left(P R_{P}\right) \subset r T \subset R$; then $P R_{P}=P$, as required.

(c): Let $S=R\left[v_{1}, \cdots, v_{m}\right]$. As in the proof of (b), the conductor $J=(R: S)$ is not contained in $P$. Since $S \neq R$ without loss of generality, reasoning as in the proof of Corollary 2.6 shows that $\operatorname{rad}_{S}(J)$ is a prime ideal of $S$ which properly contains $P R_{P} \cap S$. Thus, $\operatorname{rad}_{S}(J)=N \cap S$ which contains each $v_{i}$; as each $v_{i}$ has a power in $J$, the assertion about $I$ follows from the multinomial theorem. Finally, if $N=\Sigma R v_{i}$, it follows that $S=R+N$ is divided (since its only primes $0 \subset P R_{P} \subset N$ are divided in $T$ ), integral, (unibranched,) and module-finite over $R$. Apply (b) to complete the proof.

REMARK 2.11. It is interesting to note that Theorem 2.5 may be used in conjunction with Lemma 2.2 in order to obtain the "going-down" analogues of the "divided" assertions in Lemma 2.2. The method being similar in all cases, we provide details only for the analogue of (c); viz., if $R$ is $G D$ and $P \in \operatorname{Spec}(R)$, then $R / P$ is $G D$. A proof for the quasi-local case proceeds by taking a divided integral (unibranched) extension $T$ of $R$, and considering the prime $Q$ of $T$ which contracts to $P$. The extension $R / P \subset T / Q$ is evidently integral (and unibranched); by Lemma $2.2(\mathrm{c}), T / Q$ is also divided, and so an application of Theorem 2.5 establishes the quasi-local case. (The usefulness of condition (c) in Theorem 2.5 is apparent from the preceding argument, since $T / Q$ need not be an overring of $R / P$ even if $T$ is an overring of $R$.) As being $G D$ is a local property, the general case follows immediately from the observation that $(R / Q)_{P / Q} \cong$ $R_{P} / Q R_{P}$ for primes $Q \subset P$ of $R$ [4, Proposition 11, p. 90].

The result proved above, i.e., that any factor domain of a goingdown ring $R$ is itself a going-down ring, was established by Papick [15, Proposition 2.1] under the extra assumption that each overring of $R$ is treed, a nontrivial restriction in view of the proof of [5, Theorem 4.2(ii)]. Our general result now extends [15, Corollary 
3.19] to the assertion that any factor domain of an open ring is itself open; similarly, one may now strengthen the characterization of propen not open domains in [15, Theorem 5.18].

3. An external characterization. This section introduces a characterization of going-down rings which supplies alternate approaches to Proposition 2.1 and some of the results in Remark 2.11.

Proposition 3.1. The domain $R$ is not a going-down ring if and only if there exist $P \in \operatorname{Spec}(R)$ and a valuation overring $V$ of $R$ such that: (1) for all nonzero $v \in V, v^{-1} \notin P$, and (2) for some $w \in V, w^{-1} \in P R_{P}$.

Proof. If $R$ is not $G D$, then [6, Theorem 1] supplies a valuation overring $W$ of $R$ such that $R \subset W$ does not satisfy going-down. By the criterion for going-down [11, Exercise 37(iii), p. 44] which was used in the proof of Proposition 2.1, we obtain $P \in \operatorname{Spec}(R), Q$ minimal amongst primes of $W$ containing $P$, and an element $b$ of $P W \cap(R \backslash P)(W \backslash Q)$. Write $b=\Sigma p_{i} v_{i}$, with $p_{i}$ in $P$ and $v_{i}$ in $W$. As the ideals $W p_{i}$ are linearly ordered by inclusion, $b=p v$, with $p$ in $P$ and $v$ in $W$; also, by choice of $b, p v=r z$ for some $r$ in $R \backslash P$ and $z$ in $W \backslash Q$. Taking $V=W_{Q}$ and $w=v z^{-1}$ then guarantees (1) and (2).

Conversely, let $P$ and $V$ satisfy (1) and (2). By the above argument about linearly ordered ideals, (1) implies that $1 \notin P V$, so that there is a prime $Q$ of $V$ which is minimal over $P V$. Then (2), in conjunction with [11, Exercise 37(iii), p. 44], implies that $R \subset V$ fails to satisfy going-down, whence $R$ is not $G D$.

REMARK 3.2(a). Evidently, Proposition 3.1 provides a new proof that any divided domain is $G D$. It also seems worthwhile to indicate how Proposition 3.1 leads to a proof of the principal result of Remark 2.11, viz., that factor domains inherit the $G D$ property. (Yet another proof will be sketched in (b) below, as an application of base-change.) Indeed, if the domain $R / P$ is not $G D$, there exist a prime $Q$ of $R$ containing $P$ and a subring $T$ of $R_{P}$ containing $R+P R_{P}$, such that the prime $Q / P$ and the valuation overring $T / P R_{P}$ of $R / P$ satisfy conditions given by Proposition 3.1. Since $(Q / P)(R / P)_{Q / P}$ may be identified with $Q R_{Q} / P R_{Q}$, we may use the analogue of condition (2) while working inside $R_{P}$ to obtain: $t$ in $T \backslash P R_{P}, r$ in $Q$ and $z$ in $R \backslash Q$ such that $u=t\left(r z^{-1}\right)-1 \in P R_{P}$. As in the proof of Proposition 3.1, the analogue of condition (1) implies that $\left(Q+P R_{P}\right) / P R_{P}$ 
survives in $T / P R_{P}$, and hence yields $J$, minimal amongst primes of $T$ containing $Q$. Now, $u \in J$ (noting $\left.\left(P R_{P}\right)^{2}=P\left(P R_{P}\right) \subset Q T\right)$, whence $1+u \in T \backslash J$. Since $r t=z(1+u)$, we again invoke [11, Exercise 37(iii), p. 44], to conclude that $R \subset T$ fails to satisfy goingdown, thus establishing (the contrapositive of) the required assertion.

(b) Instead of using [11, Exercise 37(iii), p. 44] in (a) to show that the overring extension $R / P \subset T / P R_{P}$ inherits going-down from $R \subset T$, one may apply [10, Remarques 3.9.2.1(ii), p. 253]. Explicitly, set $X=\operatorname{Spec}(T)$ and $Y=\operatorname{Spec}(R)$, and note that the parenthetical observation of three sentences ago serves to identify the inverse image of $Y_{1}=\operatorname{Spec}(R / P)$ under the contraction map $f: X \rightarrow Y$ as $X_{1}=\operatorname{Spec}\left(T / P R_{P}\right)$.

(c) With the notation of (a) and (b) above, it is straightforward to verify that $\operatorname{Spec}\left(T / P R_{P}\right) \rightarrow \operatorname{Spec}(R / P)$ is an open map (using the Zariski topologies) whenever $\operatorname{Spec}(T) \rightarrow \operatorname{Spec}(R)$ is open. (As in (a) and (b), the critical observation is that any prime ideal of $T$ which contains $P$ must contain $P R_{P}$.) Consequently, as (valuation) overrings suffice in testing for open domains [16, Proposition 3.10], we recover anew a fact obtained in Remark 2.11, viz., that any factor domain of an open ring is itself open.

(d) We close by observing that Proposition 3.1 admits several variants (proved similarly). In one such, condition (1) is replaced by the requirement $P V \neq V$; in another, by the stipulation that $\operatorname{rad}_{V}(P V)$ is the maximal ideal of $V$.

\section{REFERENCES}

1. T. Akiba, A note on AV-domains, Bull. Kyoto Univ. Education Ser. B, 31 (1967), $1-3$.

2. R. A. Beauregard and D. E. Dobbs, On a class of Archimedean integral domains, Canad. J. Math., 28 (1976), 365-375.

3. M. B. Boisen, Jr. and P. B. Sheldon, Pre-Prüfer rings, Pacific J. Math. 58 (1975), 331-344.

4. N. Bourbaki, Algèbre commutative (Chaps. 1-2), Act. scient. et ind. 1290, Hermann, Paris, 1962.

5. D. E. Dobbs, On going down for simple overrings, II, Comm. in Algebra, 1 (1974), 439-458.

6. D. E. Dobbs and I. J. Papick, On going down for simple overrings, III, Proc. Amer. Math. Soc., 54 (1976), 35-38.

7. - Ascent and descent of going-down rings for integral extensions, Bull. Austral, Math. Soc., 15 (1976), 254-264.

8. R. Gilmer, Multiplicative ideal theory, Queen's Papers in Pure and Appl. Math., No. 12, Queen's University, Kingston, Ontario, 1968.

9. B. Greenberg, Global dimension of Cartesian squares, J. Algebra 32 (1974), 31-43. 10. A. Grothendieck and J. A. Dieudonné, Eléments de géométrie algébrique I, SpringerVerlag, Berlin, 1971.

11. I. Kaplansky, Commutative rings, Allyn and Bacon, Boston, 1970.

12. T. Kikuchi, Some remarks on S-domains, J. Math. Kyoto Univ., 6 (1966), 49-60. 
13. S. McAdam, Going down, Duke Math J. 39 (1972), 633-636.

14. - Simple going down, J. London Math. Soc., 13 (1976), 167-173.

15. I. J. Papick, Topologically defined classes of going-down domains, Trans. Amer. Math. Soc., 219 (1976), 1-37.

16. - dissertation, Rutgers University, New Brunswick, N. J., 1975.

17. P. B. Sheldon, How changing $D[[x]]$ changes its quotient field, Trans. Amer. Math. Soc. 159 (1971), 223-244.

Received May, 11, 1976.

UNIVERSity OF TENNESSEE 



\section{PACIFIC JOURNAL OF MATHEMATICS}

\section{EDITORS}

RICHARD ARENS (Managing Editor)

University of California

Los Angeles, California 90024

\section{J. DugunduI}

Department of Mathematics University of Southern California Los Angeles, California 90007

D. Gilbarg and J. Milgram

Stanford University

Stanford, California 94305

\author{
R. A. Beaumont \\ University of Washington \\ Seattle, Washington 98105
}

\section{ASSOCIATE EDITORS}
B. H. NeUmanN
F. WOLF
K. YosHIDA

\section{SUPPORTING INSTITUTIONS}

\author{
UNIVERSITY OF BRITISH COLUMBIA \\ CALIFORNIA INSTITUTE OF TECHNOLOGY \\ UNIVERSITY OF CALIFORNIA \\ MONTANA STATE UNIVERSITY \\ UNIVERSITY OF NEVADA \\ NEW MEXICO STATE UNIVERSITY \\ OREGON STATE UNIVERSITY \\ UNIVERSITY OF OREGON \\ OSAKA UNIVERSITY
}

\author{
UNIVERSITY OF SOUTHERN CALIFORNIA \\ STANFORD UNIVERSITY \\ UNIVERSITY OF HAWAII \\ UNIVERSITY OF TOKYO \\ UNIVERSITY OF UTAH \\ WASHINGTON STATE UNIVERSITY \\ UNIVERSITY OF WASHINGTON \\ AMERICAN MATHEMATICAL SOCIETY
}

The Supporting Institutions listed above contribute to the cost of publication of this Journal, but they are not owners or publishers and have no responsibility for its content or policies.

Mathematical papers intended for publication in the Pacific Journal of Mathematics should be in typed form or offset-reproduced, (not dittoed), double spaced with large margins. Please do not use built up fractions in the text of your manuscript. You may however, use them in the displayed equations. Underline Greek letters in red, German in green, and script in blue. The first paragraph or two must be capable of being used separately as a synopsis of the entire paper. Items of the bibliography should not be cited there unless absolutely necessary, in which case they must be identified by author and Journal, rather than by item number. Manuscripts, in triplicate, may be sent to any one of the editors. Please classify according to the scheme of Math. Reviews, Index to Vol. 39. All other communications should be addressed to the managing editor, or Elaine Barth, University of California, Los Angeles, California, 90024.

The Pacific Journal of Mathematics expects the author's institution to pay page charges, and reserves the right to delay publication for nonpayment of charges in case of financial emergency.

100 reprints are provided free for each article, only if page charges have been substantially paid. Additional copies may be obtained at cost in multiples of 50 .

The Pacific Journal of Mathematics is issued monthly as of January 1966. Regular subscription rate: $\$ 72.00$ a year (6 Vols., 12 issues). Special rate: $\$ 36.00$ a year to individual members of supporting institutions.

Subscriptions, orders for back numbers, and changes of address should be sent to Pacific Journal of Mathematics, 103 Highland Boulevard, Berkeley, California, 94708.

PUBLISHED BY PACIFIC JOURNAL OF MATHEMATICS, A NON-PROFIT CORPORATION

Printed at Kokusai Bunken Insatsusha (International Academic Printing Co., Ltd.), 8-8, 3-chome, Takadanobaba, Shinjuku-ku, Tokyo 160, Japan. 


\section{Pacific Journal of Mathematics}

Patricia Andresen and Marvin David Marcus, Weyl's inequality and

quadratic forms on the Grassmannian .......................

George Bachman and Alan Sultan, Regular lattice measures: mappings and

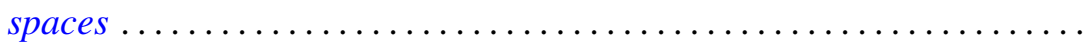

David Geoffrey Cantor, On certain algebraic integers and approximation by rational functions with integral coefficients ...................

James Richard Choike, On the value distribution of functions meromorphic in the unit disk with a spiral asymptotic value ..................

David Earl Dobbs, Divided rings and going-down................ 353

Mark Finkelstein and Robert James Whitley, Integrals of continuous

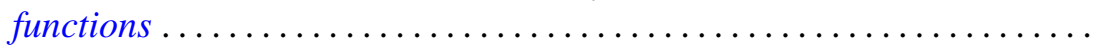

Ronald Owen Fulp and Joe Alton Marlin, Integrals of foliations on manifolds with a generalized symplectic structure ...............

Cheong Seng Hoo, Principal and induced fibrations .................

Wu-Chung Hsiang and Richard W. Sharpe, Parametrized surgery and

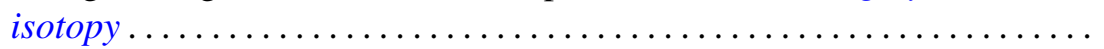

Surender Kumar Jain, Surjeet Singh and Robin Gregory Symonds, Rings whose proper cyclic modules are quasi-injective .................

Pushpa Juneja, On extreme points of the joint numerical range of commuting normal operators...

Athanassios G. Kartsatos, Nth order oscillations with middle terms of order $N-2$.

John Keith Luedeman, The generalized translational hull of a

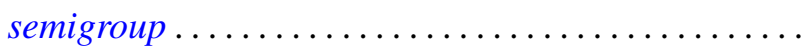

Louis Jackson Ratliff, Jr., The altitude formula and DVR's ...

Ralph Gordon Stanton, C. Sudler and Hugh C. Williams, An upper bound for the period of the simple continued fraction for $\sqrt{D}$...

David Westreich, Global analysis and periodic solutions of second order systems of nonlinear differential equations...

David Lee Armacost, Correction to: "Compactly cogenerated LCA

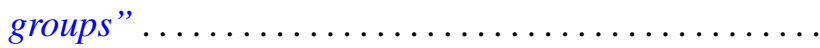

Jerry Malzan, Corrections to: "On groups with a single involution" .

David Westreich, Correction to: "Bifurcation of operator equations with unbounded linearized part" ...................... 